\title{
3D nano-structures for laser nano-manipulation
}

\author{
Gediminas Seniutinas $^{1,2}$, Lorenzo Rosa ${ }^{* 1,2}$, Gediminas Gervinskas ${ }^{1,2}$, \\ Etienne Brasselet ${ }^{3}$ and Saulius Juodkazis ${ }^{1,2}$
}

\section{Full Research Paper}

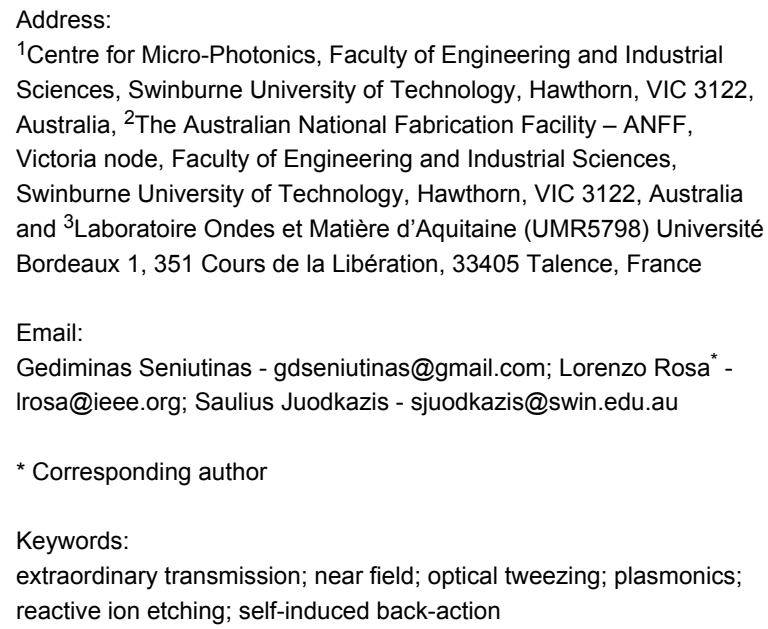

${ }^{1}$ Centre for Micro-Photonics, Faculty of Engineering and Industrial Sciences, Swinburne University of Technology, Hawthorn, VIC 3122, Australia, ${ }^{2}$ The Australian National Fabrication Facility - ANFF, Victoria node, Faculty of Engineering and Industrial Sciences, Swinburne University of Technology, Hawthorn, VIC 3122, Australia and ${ }^{3}$ Laboratoire Ondes et Matière d'Aquitaine (UMR5798) Université Bordeaux 1, 351 Cours de la Libération, 33405 Talence, France

\begin{abstract}
The resputtering of gold films from nano-holes defined in a sacrificial PMMA mask, which was made by electron beam lithography, was carried out with a dry plasma etching tool in order to form well-like structures with a high aspect ratio (height/width $\approx 3-4$ ) at the rims of the nano-holes. The extraordinary transmission through the patterns of such nano-wells was investigated experimentally and numerically. By doing numerical simulations of 50-nm and 100-nm diameter polystyrene beads in water and air, we show the potential of such patterns for self-induced back-action (SIBA) trapping. The best trapping conditions were found to be a trapping force of $2 \mathrm{pN} / \mathrm{W} / \mu \mathrm{m}^{2}$ (numerical result) exerted on a $50-\mathrm{nm}$ diameter bead in water. The simulations were based on the analytical Lorentz force model.
\end{abstract}

\section{Introduction}

Optical trapping is a fundamental experimental technique for physics and biology, which allows to precisely control and position micrometer-sized objects such as dielectric parts for nanoassembly, and biomaterials such as cells and bacteria, through the use of gradient forces, which originate from the interaction with a focused laser beam [1].
Nano-focusing and light control, which are possible with metallic plasmonic structures, are very attractive to engineer optical traps, in order to accurately position and manipulate objects down to the nanometer-scale [2]. Plasmonic nanoantennas have been designed for the trapping of dielectric and metallic particles in the size range of $10 \mathrm{~nm}$ [3-5], while fishnet 
metamaterials have been shown to optically pull 100-nm dielectric beads in simulations [6]. Double nano-hole structures have been built to trap yet smaller 12-nm objects [7], and later even objects of single-protein size [8]. When nanometer-sized objects are handled by this technique, the trapping force weakens and the trap loses stability due to Brownian motion. This forces the laser intensity to be increased up to the point where damage occurs to the delicate biomaterials.

Self-induced back-action (SIBA) [9] has recently emerged as a promising technique to address this shortcoming. The main feature of SIBA trapping is the contribution of the object in defining the optical field distribution in the trap as well as the trapping conditions through focusing/diffraction and the interaction with the localized field on the substrate surface. This opens new degrees of freedom to the device, which can be exploited to reduce the optical intensity required for trapping, and thus to prevent damage to the biological material. The trapping position can also be moved farther away from the device, which provides access to the object from all directions. This technique has been employed with photonic crystals to trap spheres in nano-cavities [10] and to control the translation and the rotation of nano-rods by using resonators etched in waveguides [11].

SIBA trapping and slot-guiding of nano-particles using plasmonic devices $[12,13]$ can be realized by using patterns of hole arrays and grooves. The extraordinary transmission in plasmonics can be exploited in metal hole arrays (MHA) at visible and IR spectral wavelengths as a promising method to introduce narrow-band wavelength-selective filtering [14]. A combination of extraordinary transmission and trapping is a promising direction for handling, moving, and sorting nano-materials. By the creation of patterns, which make plasmonic nano-particles chiral, one can envisage the creation of a controlled delivery of force and torque, hence, nano-hands. Recently, it was demonstrated that even optical pulling can be exerted using plasmonic nano-structures [15].

The fabrication of large areas filled with three-dimensional (3D) nano-structures with SIBA functionality requires a multi-step processing and is slow in throughput. Here, we show a parallel processing route for the fabrication of 3D nano-well structures over large areas of cross-sections in the sub-mm range. We explore experimentally and numerically whether extraordinary transmission [16] can be controlled in terms of intensity and spectral width using such 3D nano-well patterns, and we show the suitability of the fabricated structures for SIBA trapping.

\section{Experimental}

Gold re-sputtering was carried out for $3.5 \mathrm{~min}$ in Ar plasma (Samco RIE-101iPH) at a bias power of $200 \mathrm{~W}$ and at a process pressure of $2 \mathrm{~Pa}$. The inductively coupled plasma (ICP) etching mode was turned off. Patterns on a sacrificial 300-nm thick PMMA mask were defined by electron beam lithography (EBL; Raith $150^{\mathrm{TWO}}$ ). The mask was spin coated on a cover glass which was magnetron sputter-coated (AXXIS, JKLesker) with a $100 \mathrm{~nm}$ thick gold film. Figure 1a and Figure 1b show a sketch of the sample structure before and after the re-sputtering step, with conical well structures formed at the rim of holes in the $\mathrm{Au}$ film. The plasma etching rate of PMMA was approximately 2.0-2.5 times higher than that of Au. The opening of the holes in the Au-film and the structural quality of samples were characterized by scanning electron microscopy (SEM).

\section{Numerical simulations}

The structure was modeled as a glass substrate on which a gold layer of $100 \mathrm{~nm}$ thickness was patterned with a lattice of waterfilled $(n=1.33)$ holes of $200 \mathrm{~nm}$ diameter. The nano-wells were placed on top of the holes as $100 \mathrm{~nm}$ high cylindrical gold rings (a)

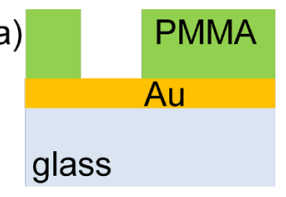

(b)

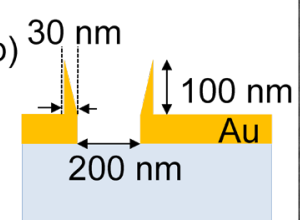

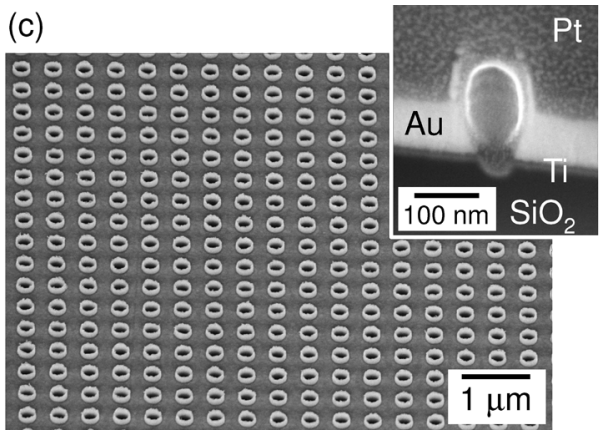

$\mathrm{Pt}$

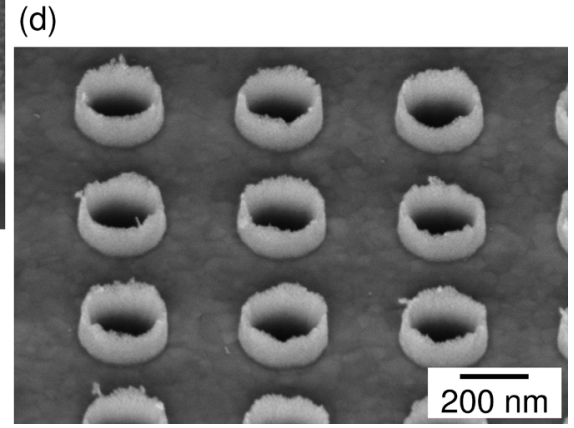

Figure 1: Illustration of the nano-well fabrication by (a) dry plasma etching of Au-film and PMMA mask, which results in Au re-sputtering and (b) formation of a nano-well around the rim of the hole. The experimental outcome is shown as (c) SEM image of the 3D Au nano-wells array; in (d) the magnified slanted view shows details of the nano-well edges. The inset in panel (c) shows the cross-section of a nano-well after deposition of a protective Pt layer. 
filled with water. Two cases were considered: one with cylindrical rings of $30 \mathrm{~nm}$ wall thickness, and one with conical rings, in which the wall thickness tapers from $30 \mathrm{~nm}$ at the bottom to zero at the top, leaving the conical shape on the inside (Figure 1b). The refractive index spectra of the materials were fitted from the experimental values obtained in literature, by means of a built-in polynomial model.

The finite-difference time-domain (FDTD) included a section of the substrate, enclosed in all directions by perfectly matched layers (PML) to avoid spurious reflections. The central area of $5 \times 5$ periods was illuminated by a total-field/scattered-field (TFSF) source, in order to measure separately the total field in the central area, and the field scattered outside and to calculate the cross-sections. For the calculation of transmitted and reflected power by the substrate, the domain was changed to a plane-wave illumination from the bottom, also modifying the lateral domain boundaries to periodic boundary conditions (PBC) to avoid diffraction of the plane wave from the borders. In this case, the reflected and transmitted power were measured by two power monitors, placed at either end of the domain, to gather evidence of extraordinary transmission. For the force calculation, the trapped object (a polystyrene bead) was introduced in the total-field region, and surrounded by a 3D monitor recording the vectorial $\mathrm{E}$ - and $\mathrm{H}$-fields, discriminating the object volume by the refractive index change, and applying the Lorentz force formulation explained in the following section. The source was linearly $x$-polarized with a bandwidth range from 400 to $1400 \mathrm{~nm}$.

The simulations were performed on the swinSTAR supercomputer at Swinburne University with 16-core nodes of $64 \mathrm{~GB}$ memory each. Each simulation took about 1 hour on a 16-node cluster with 256 total cores.

\section{Background: Lorentz force}

The Lorentz force calculation is presented below for the "bound" and "free" charges and currents as $\rho_{\mathrm{b}, \mathrm{f}}$ and $\mathbf{j}$ b,f , respectively. Let us consider a monochromatic field and consider nonmagnetic media only. Using the complex representation for the light field, the real Lorentz force density $\left[\mathrm{N} \cdot \mathrm{m}^{-3}\right]$ is:

$$
\mathbf{f}=\frac{1}{2} \operatorname{Re}\left(\rho \mathbf{E}^{*}\right)+\frac{1}{2} \operatorname{Re}\left(\mathbf{j} \times \mathbf{B}^{*}\right)=\mathbf{f}_{\mathrm{e}}+\mathbf{f}_{\mathrm{h}}
$$

where $\rho=\rho_{\mathrm{b}}+\rho_{\mathrm{f}}$ and $\mathbf{j}=\mathbf{j}_{\mathrm{b}}+\mathbf{j}_{\mathrm{f}}$ are the total charge densities.

First, let us consider the case of pure dielectrics without a free charge or a free current density. On the one hand, we have $\rho_{b}=$ $-\nabla \cdot \mathbf{P}$ and $\rho_{\mathrm{f}}=0$, hence $\nabla \cdot \mathbf{D}=0$ with $\mathbf{D}=\varepsilon_{0} \varepsilon_{\mathrm{r}} \mathbf{E}=\varepsilon_{0} \mathbf{E}+\mathbf{P}$ where $\mathbf{P}$ is the material polarization due to the bound charges and $\varepsilon_{\mathrm{r}}$ is complex if the medium is not transparent. On the other hand, we have $\mathbf{j}_{\mathrm{f}}=0, \mathbf{j}_{\mathrm{b}}=\partial_{\mathrm{t}} \mathbf{P}$, which leads to:

$$
\rho=\varepsilon_{0} \nabla \cdot \mathbf{E} ; \quad \mathbf{j}=\varepsilon_{0}\left(\varepsilon_{\mathrm{r}}-1\right) \partial_{\mathrm{t}} \mathbf{E}
$$

For materials whose dielectric permittivity accounts for conduction electrons, i.e., metals, one would have $\rho_{\mathrm{b}}=-\nabla \cdot \mathbf{P}$ and $\nabla$. $\mathbf{D}=\rho_{\mathrm{f}}$. On the other hand, $\nabla \times \mathbf{H}=\mathbf{j}_{f}+\varepsilon_{0} \varepsilon_{r} \partial_{t} \mathbf{E}$ and $\mathbf{j}_{b}=\partial_{t} \mathbf{P}=$ $\varepsilon_{0}\left(\varepsilon_{r}-1\right) \partial_{t} \mathbf{E}$. Therefore, we obtain:

$$
\rho=\varepsilon_{0} \nabla \cdot \mathbf{E} ; \quad \mathbf{j}=\nabla \times \mathbf{H}-\varepsilon_{0} \partial_{\mathrm{t}} \mathbf{E}
$$

The 3D monitor in the FDTD returns the $\mathbf{E}$ and $\mathbf{H}$ fields and we calculate $\mathbf{B}=\mu_{0} \mathbf{H}$, and, supposing linear media, $\mathbf{D}=\varepsilon_{0} \varepsilon_{\mathrm{r}} \mathbf{E}$. At the interface between metal and dielectric, the component of $\mathbf{E}$ parallel to the interface is continuous. The component of $\mathbf{D}$ perpendicular to the interface must also be continuous, which means the perpendicular component of $\mathbf{E}$ must be discontinuous and generate a charge density $\rho=\rho_{b}+\rho_{f}$.

When calculating the charge density, $\rho$, through the divergence of the electric field, one must take into account an unavoidable feature of the FDTD method in that each field component $\left(E_{x}\right.$, $\left.E_{y}, E_{z}, H_{x}, H_{y}, H_{z}\right)$ is calculated for a different point of the Yee cell. As it was recently shown [17], an interpolation scheme can be used to estimate the field at the boundary. Similarly, for the magnetic force, the cross product calculation requires to interpolate the $\mathbf{B}$ vector components in the positions where the components of $\mathbf{j}$ are defined. In this case, both $B_{x}$ and $B_{y}$ are discontinuous at the interface. However, with the employed scheme [17] the interpolation error only affects the magnetic $z$-component of the Lorentz force $\left\langle f_{z}\right\rangle=1 / 2 \operatorname{Re}\left(j_{x} B_{y}^{*}-j_{y} B_{x}^{*}\right)$.

The total force per unit volume $\langle\mathbf{f}\rangle=\left\langle\mathbf{f}_{\mathrm{e}}\right\rangle+\left\langle\mathbf{f}_{\mathrm{h}}\right\rangle$ is then integrated on the trapped object volume $\mathcal{V}$ obtaining

$$
\mathbf{F}=\iiint_{\mathcal{V}}\langle\mathbf{f}\rangle \mathrm{d} v
$$

The Maxwell stress tensor (MST) approach can also be used for force calculation, however, there is an ambiguity in the placement of the monitors across the interfaces [17]. In case of the method outlined here, the 3D field monitors, which capture the complex values of the fields provide direct means to remove the ambiguity and calculate the Lorentz force. The analytical formulas presented here were implemented in the simulations. 


\section{Results and Discussion Optical transmission}

Figure 1c and Figure 1d show SEM images of the fabricated structures. Following the usual PMMA resist development procedure after dry etching, the formation of uniform well-like structures was observed at the rim of the etched holes. The structures were approximately $100 \mathrm{~nm}$ in height and about $20-30 \mathrm{~nm}$ in width at the base. Their formation can be understood as a re-deposition of the sputtered gold film. During sputtering some gold is deposited on the inner walls of the openings in the sacrificial PMMA layer. This results in a free-standing pattern with enough structural strength to withstand the wetbath development. A lengthy one-hour treatment at $70{ }^{\circ} \mathrm{C}$ in an ultrasonic bath would be necessary to remove these nano-well structures. The wells are made of $\mathrm{Au}$ as confirmed by using energy dispersive X-ray spectroscopy using tilted slices, as shown in the inset of Figure 1c.
Figure 2a compares transmission and reflectivity spectra of the hole array structure with cylindrical and conical nano-well patterns. The major spectral features are similar, and by tuning the geometrical parameters of the pattern it is possible to maximize the transmission of specific wavelengths. Manifestation of extraordinary transmission can be seen at the major transmission peak around $830 \mathrm{~nm}$. The classical Bethe transmission ratio through a hole of radius $D / 2$ in an opaque screen would follow a $T \propto[(D / 2) / \lambda]^{4}$ scaling; for $D=200 \mathrm{~nm}$ and $\lambda=$ $800 \mathrm{~nm}$ it would be $T \approx 0.02 \%$. By adding the nano-well structure, the spectral properties are not affected strongly and the transmission is reduced. The extinction cross-sections $\sigma_{\text {ext }}=$ $\sigma_{\text {abs }}+\sigma_{\text {scat }}$ defined by the sum of absorption and scattering are shown in Figure 2b. The wavelengths of 760 and $808 \mathrm{~nm}$ are chosen for analysis of the light intensity distribution and force mapping (discussed below) because there is no strong pushing (scattering force), which exists at the extraordinary transmis-
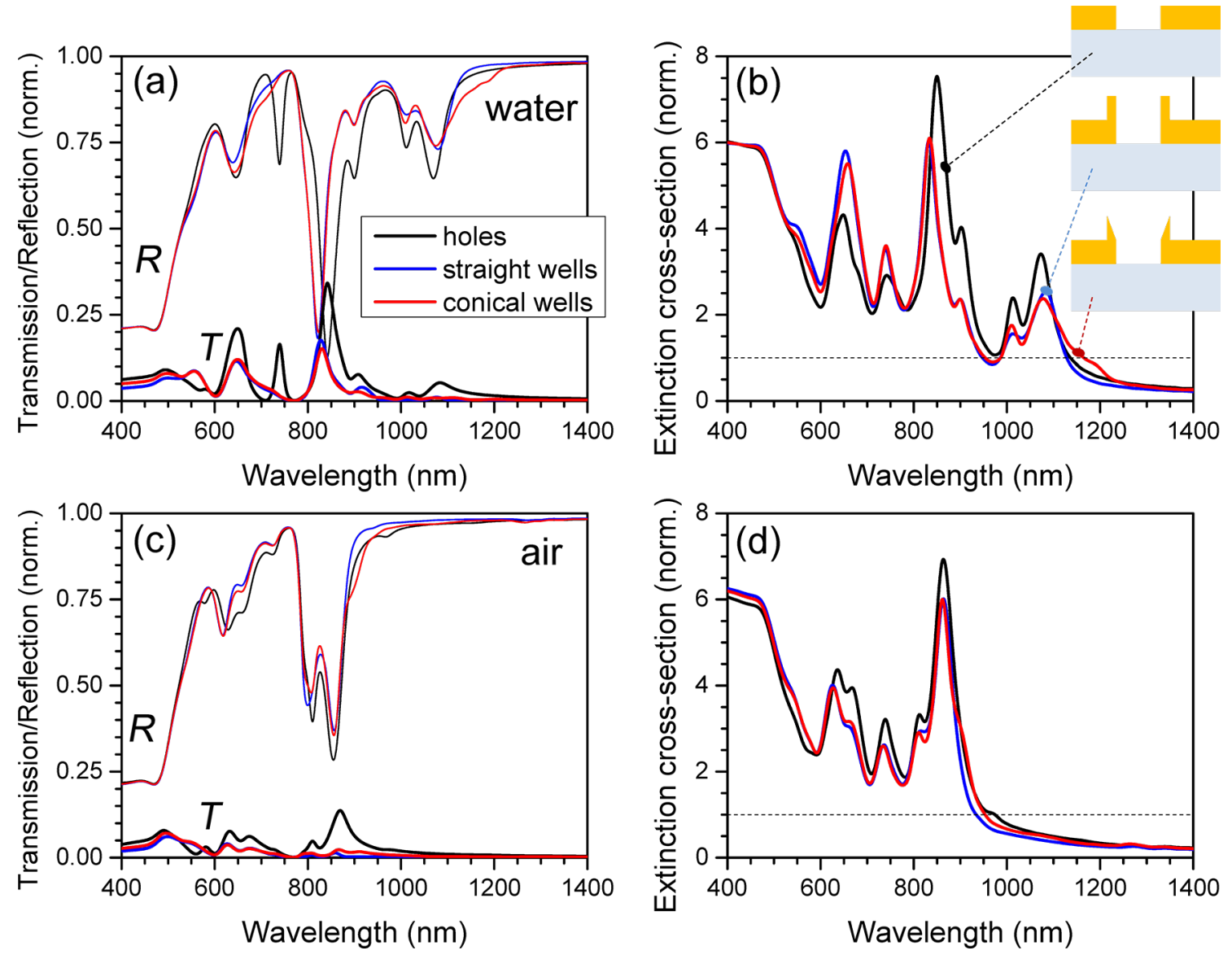

Figure 2: (a) Transmission $T$ and reflectivity $R$ coefficients for different geometries of the hole/well-array structures (see Figure 1 for geometry: hole diameter $D=200 \mathrm{~nm}$, well height $h=100 \mathrm{~nm}$, width of the well structure at the base $w=30 \mathrm{~nm}$, and thickness of Au film $100 \mathrm{~nm}$ ). Calculated for immersion in water with refractive index $n_{\mathrm{w}}=1.33$. (b) Extinction cross-sections normalized to the geometrical cross section (dashed line). (c) and (d) are the same as (a) and (b), but for air. 
sion maxima. Also, these are the wavelengths for the planned future laser trapping experiments.

The transmission of the nano-well arrays was experimentally characterized for hole diameters of 70, 90, and $120 \mathrm{~nm}$, as shown in Figure 3. The extinction, Ext, was calculated as Ext $=$ $\ln \left(T_{\text {ref }} / T_{\text {sample }}\right)$, where $T_{\text {ref }}$ is the transmissivity of a $100-\mathrm{nm} \mathrm{Au}$ layer. When the diameter is increased, the extinction peak is red-shifted and becomes negative, which corresponds to extraordinary transmission through the array, which in case of the $120-\mathrm{nm}$ array extends over a wide band between 500 and $730 \mathrm{~nm}$. The spectral tunability of the extinction in the plasmonic band around $600 \mathrm{~nm}$ is clearly discernable, and makes it possible to tune the peak up to the laser wavelength to optimize the trapping effect.

The light intensity distributions in the top-illuminated substrates reveals the configuration of the trapping field: while the field enhancement is not very strong (less than 10) in water, as shown in Figure 4a and Figure 4b, we see the formation of large spots hovering above the nano-well apertures: These spots vary little with the wavelength and are useful for trapping dielectric objects. In air the enhancement is much weaker, as shown in Figure $4 \mathrm{c}$, and at the longer wavelength in Figure $4 \mathrm{~d}$ there occurs the formation of hot-spots at the upper and lower corner of the nano-wells.

Next, we employed the full-3D vectorial model to numerically determine the optical trapping force $\mathbf{F}(\mathbf{r}, \omega)=$ $1 / 2\left[\alpha^{\prime}(\omega)+i \alpha^{\prime \prime}(\omega)\right] \nabla \mathbf{E}(\mathbf{r}, \omega)^{2}$ experienced by the bead, which is usually approximated in theory as an optical dipole oscillator, in the vicinity of a nano-well, where $\left(\alpha^{\prime}+i \alpha^{\prime \prime}\right)$ is the polariz- ability at the angular frequency $\omega$ (linked to the permittivities through the Clausius-Mossotti equation [18]), $\mathbf{r}$ is the position vector, and $\nabla \mathbf{E}^{2}$ is the E-field intensity gradient [19]. For stable trapping, the force given by the light intensity gradient (proportional to $\alpha^{\prime}$ ) must exceed the force resulting from the momentum transfer by the photons, which are back-scattered by the particle (proportional to $\alpha^{\prime \prime}$ ), and generate a potential minimum low enough to curb the combined effect of gravity, buoyancy, and the Brownian motion of the particle [20]. The polarizability of the nano-materials determines the sign of the force: metallic-like particles are repelled from high intensity regions, while dielectric nano-particles will be attracted.

\section{Force mapping}

The force mapping was calculated by using the Lorentz force formalism (from section "Background: Lorentz force") on a polystyrene-bead probe $(n=1.504)$ of diameter $d$ with the 3D-FDTD method. In order to build the map, the bead is moved in 10-nm steps along the $x z$-plane at different heights in the proximity of the top of the well. At each position the simulation is repeated and the 3D Lorentz force is calculated. Due to the density of polystyrene being approximately equal to that of water, buoyancy and gravity almost compensate each other, leaving a residual force about three orders of magnitude lower than the Lorentz force under normal illumination conditions. Figure 5 and Figure 6 show the force field map at the rim of the nano-well.

The best trapping conditions were found for the conical nanowells at $760 \mathrm{~nm}$ wavelength. The trapping locations depicted by arrow plots in Figure 5 for a bead with $d=50 \mathrm{~nm}$ and Figure 6 for a bead with $d=100 \mathrm{~nm}$, show very different equilibria

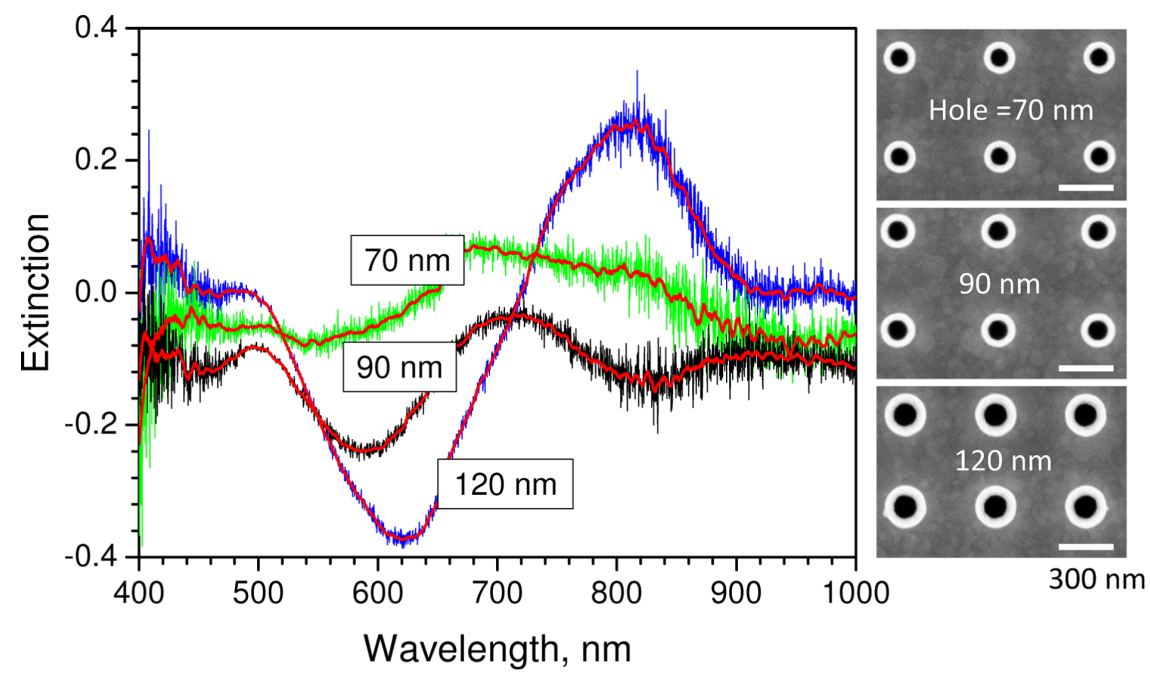

Figure 3: Experimentally measured extinction of the nano-well substrate for hole diameters of 70,90 , and $120 \mathrm{~nm}$. The value is normalized to a continuous 100-nm Au layer, thus the negative regions offer proof of extraordinary transmission [16] through the array. 


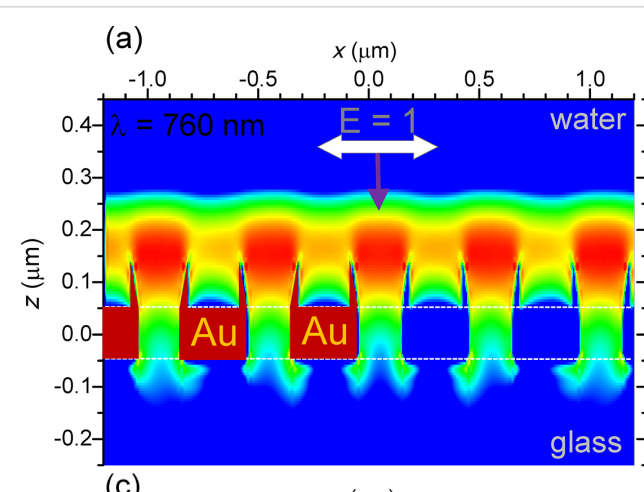

(b)

$x(\mu \mathrm{m})$
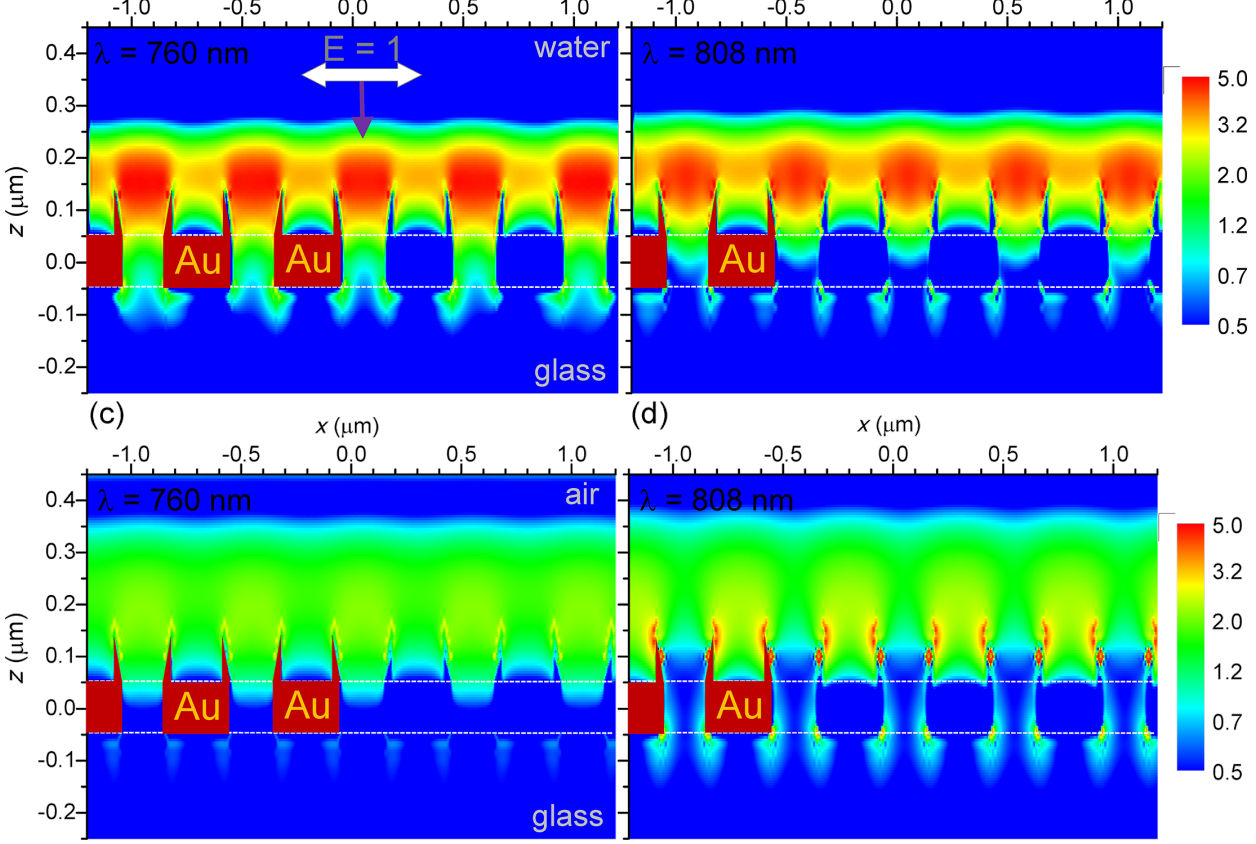

(d) $\quad x(\mu \mathrm{m})$

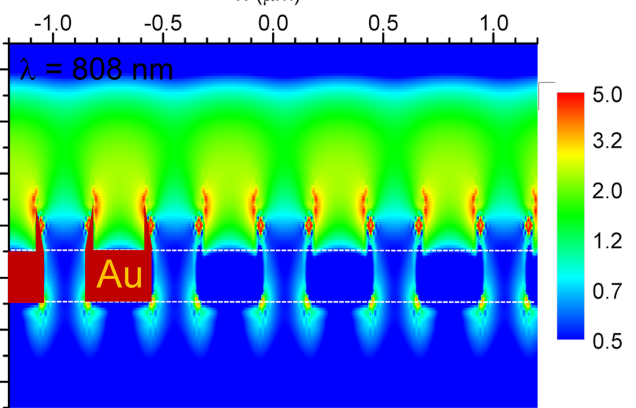

Figure 4: Light intensity distribution in the $x z$-plane simulated by 3D-FDTD at the two laser wavelengths $(a, c) 760 \mathrm{~nm}$ and $(\mathrm{b}, \mathrm{d}) 808 \mathrm{~nm}$, in $(\mathrm{a}, \mathrm{b})$ water and $(c, d)$ air. Incident light intensity $\left|E_{0}\right|^{2}=1$; propagation in negative $z$-direction.

(a)

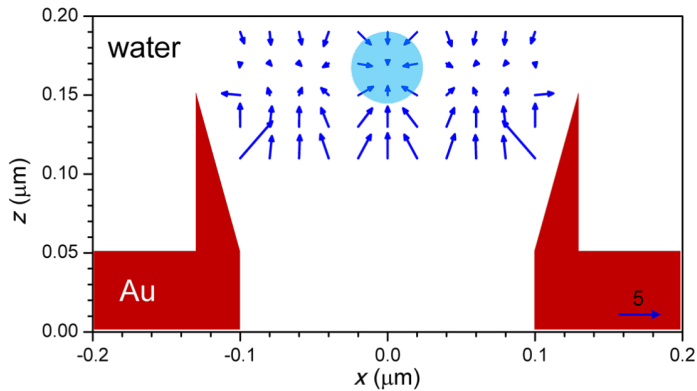

(b)

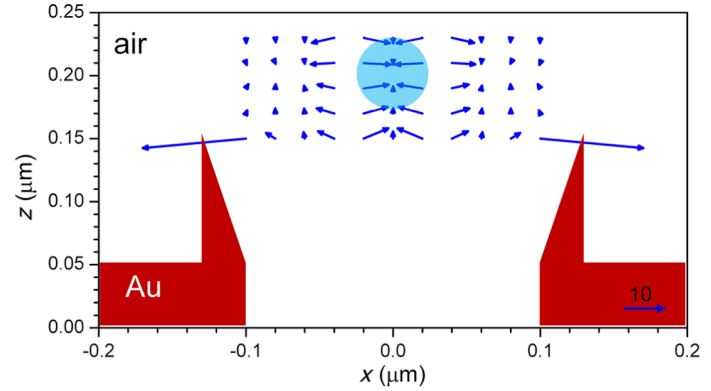

Figure 5: Arrow plot of the trapping force for plane-wave illumination in medium-to-substrate direction (as in Figure 4) at $760 \mathrm{~nm}$ wavelength for a 50-nm diameter polystyrene bead in (a) water and (b) air. The conical Au-well profile is schematically shown by the triangular shapes and the circle indicates the position of the bead in the central trap. The scale arrows at the lower right indicate forces of 5 and $10 \mathrm{pN} / \mathrm{W} / \mu \mathrm{m}^{2}$.
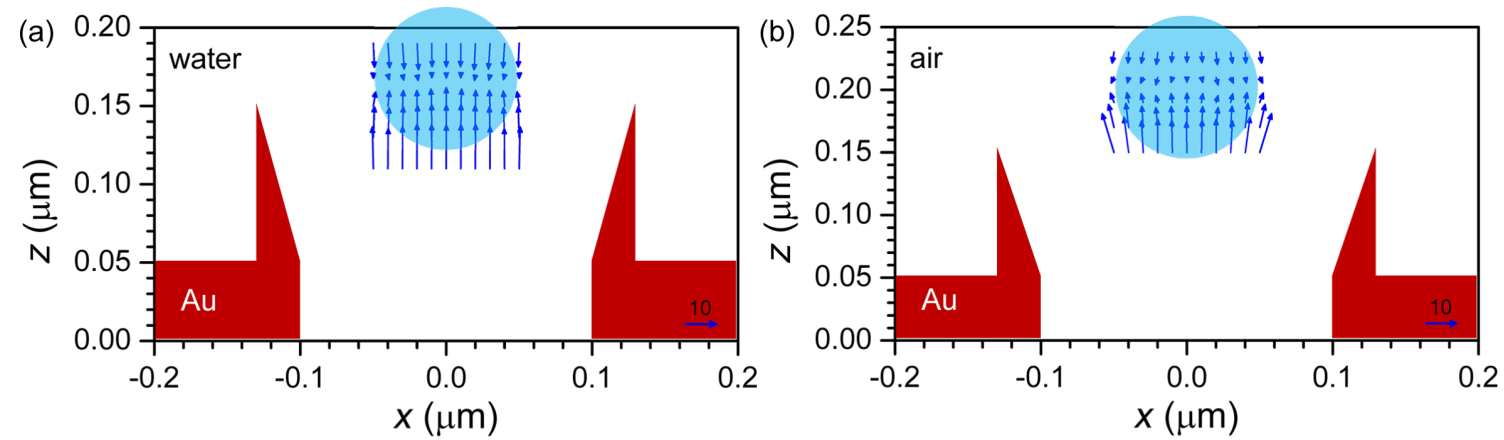

Figure 6: Arrow plot of the trapping force as in Figure 5 at $760 \mathrm{~nm}$ wavelength for a 100-nm diameter bead in (a) water and (b) air. The scale arrows at the lower right indicate a force of $10 \mathrm{pN} / \mathrm{W} / \mu^{2}$. 
between the transverse force $f_{x}$ and the longitudinal force $f_{z}$. In all cases, there is a central trapping spot at $\left(0, z_{0}\right)$, while the $50-\mathrm{nm}$ bead also shows lateral ones (making a ring at about $50 \mathrm{~nm}$ distance from the center) with weaker transverse confinement and a tendency to easily escape the nano-well. This provides a strategy for moving the particles from one well to the neighboring ones by 'storing' them in the ring. The trapping height $z_{0}$ of about $165 \mathrm{~nm}$ in water and about $200 \mathrm{~nm}$ in air, measured from the center of the metal layer, is relatively independent of the particle size. However, due to the particle altering the field distribution, the force balance is strongly affected.

The most balanced trapping is achieved in water for the $50-\mathrm{nm}$ particle in Figure 5a, with a maximum force of $2 \mathrm{pN} / \mathrm{W} / \mu \mathrm{m}^{2}$ in all directions in the particle vicinity. In air (Figure 5b) the longitudinal confinement is similar, but the lateral confinement is much stronger at $5 \mathrm{pN} / \mathrm{W} / \mu \mathrm{m}^{2}$, which makes it more difficult to shift the particle between neighboring wells. In the transverse direction, the smaller particle shows the escape distance from the central trap to be around half of the diameter, with the maximum force occurring at the maximum field gradient, which is consistent with the behavior of a single-beam optical tweezer [21]. One would then expect this distance to be independent of the diameter, as the particle size is much smaller than the wavelength. However for the 100-nm particle in Figure 6 we have a very strong longitudinal force $f_{z}$ of more than $20 \mathrm{pN} / \mathrm{W} /$ $\mu \mathrm{m}^{2}$, but a very weak lateral confinement as the dielectric particle is attracted by the hot-spots along the nano-well walls. Because of this, the particle will tend to move randomly across the well, eventually escaping it in the case of air, while for water-trapping the lower position permits confinement by the walls, as long as the mechanical strength of the metal cone is enough to resist the impact of the particle. The increase in $z$-force with the diameter is also consistent with single-beam tweezers, where the maximal force scales with the bead volume for particles, which are small with respect to the wavelength [20].

This is confirmed by examining the force components in the trap to calculate the stiffness around the trapping spot [22] as shown in Figure 7. Considering an incident intensity of $1 \mathrm{~W} / \mu \mathrm{m}^{2}$, for the longitudinal $z$-direction we see that for the $50-\mathrm{nm}$ bead in Figure $7 \mathrm{a}$, the trap has a stiffness of about $0.06 \mathrm{pN} / \mathrm{nm}$ in water and $0.04 \mathrm{pN} / \mathrm{nm}$ in air. In the transverse $x$-direction in Figure $7 \mathrm{~b}$, the stiffness is about $0.05 \mathrm{pN} / \mathrm{nm}$ in water and $0.25 \mathrm{pN} / \mathrm{nm}$ in air for the central spot. Because the diameter is small with respect to the wavelength, the stiffness is expected to increase with the diameter, as the amount of material intercepted by the field is larger, which increases the trap-
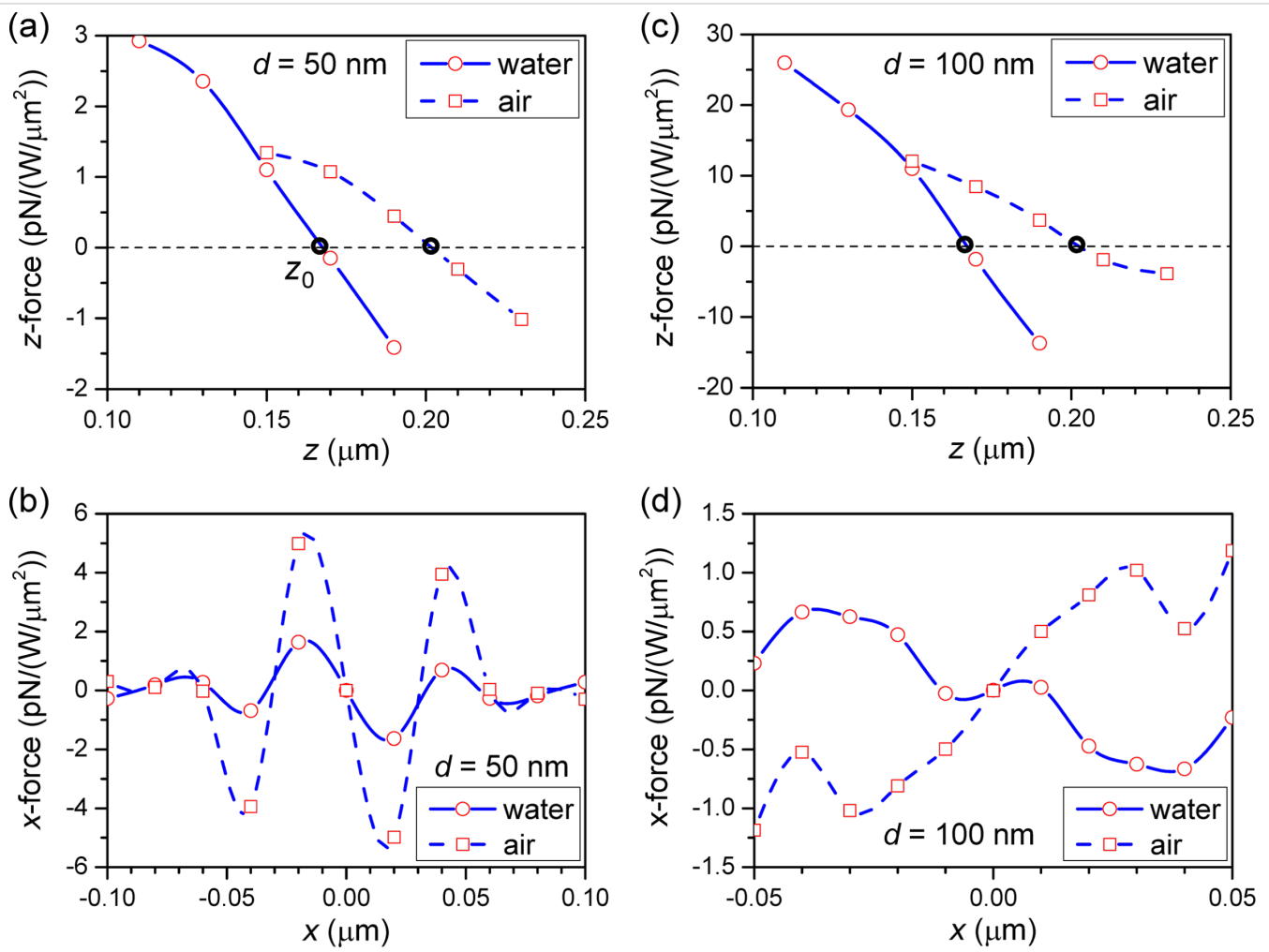

Figure 7: Force evaluation in the neighborhood of the central trapping spot $\left(0, z_{0}\right)$ at $760 \mathrm{~nm}$ wavelength for a bead of diameter $d$ in water (solid) and air (dashed): $(a, c) f_{z}(z)$ at $x=0$ and $(b, d) f_{x}(x)$ at $z=z_{0}$. The calculated values are indicated by the red markers, the blue lines are guides to the eye. 
ping power [21]. However, due to the close SIBA interaction and redistribution of field, the $z$-direction stiffness increases by about one order of magnitude for the 100-nm bead in Figure 7c, while the transverse confinement is very different in Figure $7 d$ at about $0.01 \mathrm{pN} / \mathrm{nm}$ in water. In air we notice that the particle is actually pushed away from the center and tends to escape the well, except for a weak confinement ring at a distance of $40 \mathrm{~nm}$ from the center.

Because the trapping position is relatively far away from strong field gradients, the escape force in the nano-wells is about one hundredth, and the stiffness is about one tenth, in comparison to the original SIBA trapping-substrate [9]. Yet, they are still significantly stronger than the maximum force given by Brownian motion. However, there is a significant benefit in that the nano-wells trap the particle at a much more exposed position, which makes the particle accessible from all sides to inspection and imaging. In addition the distance from the substrate makes it easier to probe the particle without interfering with the trap.

\section{Conclusions}

We demonstrate a simple plasma etching procedure which produces conical nano-well structures. The potential use in laser-trapping through the SIBA mechanism is numerically corroborated and analytical formulas are presented. The complex extinction spectra of the structures show that wavelength tunable lasers would be the best candidates to explore the peculiarities of nano-tweezing. We can foresee an enhancement of nano-material delivery into regions of high light intensity via Marangoni flow induced by localized heating and convection [23].

\section{Acknowledgements}

Part of this work was performed on the swinSTAR supercomputer at Swinburne University of Technology. Author contributions listed: E. B. and S. J. originated the idea and L. R. implemented the numerical models; G. G. and G. S. fabricated samples and characterized them. All the authors contributed to the data analysis through discussions and drafted/edited the manuscript. G. S. and L. R. contributed equally to this work.

\section{References}

1. Ashkin, A.; Dziedzic, J. M.; Bjorkholm, J. E.; Chu, S. Opt. Lett. 1986, 11, 288-290. doi:10.1364/OL.11.000288

2. Juan, M. L.; Righini, M.; Quidant, R. Nat. Photonics 2011, 5, 349-356. doi:10.1038/nphoton.2011.56

3. Zhang, W.; Huang, L.; Santschi, C.; Martin, O. J. F. Nano Lett. 2010, 10, 1006-1011. doi:10.1021/nl904168f

4. Kohoutek, J.; Dey, D.; Bonakdar, A.; Gelfand, R.; Sklar, A.; Memis, O. G.; Mohseni, H. Nano Lett. 2011, 11, 3378-3382. doi:10.1021/nl201780y
5. Kang, J.-H.; Kim, K.; Ee, H.-S.; Lee, Y.-H.; Yoon, T.-Y.; Seo, M.-K.; Park, H.-G. Nat. Commun. 2011, 2, No. 582. doi:10.1038/ncomms1592

6. Cao, T.; Zhang, L.; Cryan, M. J. Photonics J., IEEE 2012, 4, 1861-1869. doi:10.1109/JPHOT.2012.2218589

7. Pang, Y.; Gordon, R. Nano Lett. 2011, 11, 3763-3767. doi:10.1021/nl201807z

8. Pang, Y.; Gordon, R. Nano Lett. 2012, 12, 402-406. doi:10.1021/nl203719v

9. Juan, M. L.; Gordon, R.; Pang, Y.; Eftekhari, F.; Quidant, R. Nat. Phys. 2009, 5, 915-919. doi:10.1038/nphys1422

10. Descharmes, N.; Dharanipathy, U. P.; Diao, Z.; Tonin, M.; Houdré, R. Phys. Rev. Lett. 2013, 110, 123601. doi:10.1103/PhysRevLett.110.123601

11. Kang, P.; Serey, X.; Chen, Y.-F.; Erickson, D. Nano Lett. 2012, 12, 6400-6407. doi:10.1021/nl303747n

12. Yang, A. H. J.; Moore, S. D.; Schmidt, B. S.; Klug, M.; Lipson, M.; Erickson, D. Nat. Phys. 2009, 457, 71-75. doi:10.1038/nature07593

13. Chen, C.; Juan, M. L.; Li, Y.; Maes, G.; Borghs, G.; Van Dorpe, P.; Quidant, R. Nano Lett. 2012, 12, 125-132. doi:10.1021/nl2031458

14. Nishijima, Y.; Nigorinuma, H.; Rosa, L.; Juodkazis, S. Opt. Mater. Express 2012, 2, 1367-1377. doi:10.1364/OME.2.001367

15. Dogariu, A.; Sukhov, S.; Sáenz, J. Nat. Photonics 2013, 7, $24-27$. doi:10.1038/nphoton.2012.315

16. Ebbesen, T. W.; Lezec, H. J.; Ghaemi, H. F.; Thio, T.; Wolff, P. A. Nature 1998, 391, 667-669. doi:10.1038/35570

17. Gervinskas, G.; Rosa, L.; Brasselet, E.; Juodkazis, S. Proc. SPIE 2013, 8613, 861304. doi:10.1117/12.2002281

18. Jackson, J. D. Classical Electrodynamics, 3rd ed.; John Wiley \& Sons: New York, NY, 1998.

19. Ramanandan, G.; Dharmadhikari, A. K.; Dharmadhikari, J. A.; Ramachandran, H.; Mathur, D. Opt. Express 2009, 17, 9614-9619. doi:10.1364/OE.17.009614

20. Agayan, R. R.; Gittes, F.; Kopelman, R.; Schmidt, C. F. Appl. Opt. 2002, 41, 2318-2327. doi:10.1364/AO.41.002318

21. Ashkin, A. Biophys. J. 1992, 61, 569-582. doi:10.1016/S0006-3495(92)81860-X

22. Simmons, R. M.; Finer, J. T.; Chu, S.; Spudich, J. A. Biophys. J. 1996, 70, 1813-1822. doi:10.1016/S0006-3495(96)79746-1

23. Louchev, O. A.; Juodkazis, S.; Murazawa, N.; Wada, S.; Misawa, H. Opt. Express 2008, 16, 5673-5680. doi:10.1364/OE.16.005673

\section{License and Terms}

This is an Open Access article under the terms of the Creative Commons Attribution License (http://creativecommons.org/licenses/by/2.0), which permits unrestricted use, distribution, and reproduction in any medium, provided the original work is properly cited.

The license is subject to the Beilstein Journal of Nanotechnology terms and conditions: (http://www.beilstein-journals.org/bjnano)

The definitive version of this article is the electronic one which can be found at:

doi:10.3762/bjnano.4.62 